\title{
Perfil clínico e sociodemográfico de pacientes hemodialisados
}

\section{Clinical and socio-demographic profile of hemodialysis patients}

\author{
Francisco das Chagas Araújo Sousa' $\bullet$ Vaniele Sousa Carvalho Resende ${ }^{2} \bullet$ Wenderson Costa da Silva $^{3}$ \\ Pedro Gabriel Sobral da Silva ${ }^{4}$ Brunna Matos Sousa ${ }^{5}$ Bianca Santos Soares $^{6} \bullet$ Rogério Cruz Mendes $^{7}$ \\ Eduardo Brito da Silva ${ }^{8} \bullet$ Wallison Hamon Silva Lima ${ }^{9}$ Maria Gabrielle Sobral da Silva ${ }^{10}$ \\ Amarildo Barbosa da Costa" $\bullet$ Lisianne Natália Santos Silva ${ }^{12}$
}

\begin{abstract}
RESUMO
Objetivo: descrever o perfil clínico e sociodemográfico dos pacientes hemodialisados. Método:Trata-se de um estudo descritivo, exploratório, com abordagem quantitativa, realizada em um centro de hemodiálise em Teresina-PI, através de um questionário realizado com 50 pacientes. Resultados: quanto aos resultados $40 \%$ eram do sexo masculino com faixa etária entre 48 a 57 anos e 58 a 67 anos; cor da pele pardo com 72\%; 66\% eram casados; $78 \%$ aposentados; $82 \%$ com renda familiar de até I salário mínimo; 44\% com procedência de Teresina; e 34\% com ensino fundamental incompleto. $O$ tempo de tratamento de hemodiálise eram de 5 a 9 anos com 52\% a principal doença de base foi a Hipertensão Arterial Sistêmica (HAS). Sobre a importância da dialise todos consideram importante o tratamento. Conclusão: Esses dados ajudaram os profissionais de saúde a conhecer o perfil dos pacientes para auxílio do planejamento de uma assistência direcionada à prevenção e também para melhorar a expectativa de vida dos que são submetidos a hemodiálise.
\end{abstract}

Palavras-chave: Diálise Renal; Doença Renal Crônica; Perfil de Saúde.

\begin{abstract}
Objective: to describe the clinical and sociodemographic profile of hemodialysis patients. Method:This is a descriptive, exploratory study with a quantitative approach, carried out at a hemodialysis center in Teresina-PI, using a questionnaire with 50 patients. Results: as for the results, $40 \%$ were male, aged between 48 to 57 years and 58 to 67 years; brown skin color with $72 \% ; 66 \%$ were married; $78 \%$ retired; $82 \%$ with a family income of up to I minimum wage; $44 \%$ from Teresina; and $34 \%$ with incomplete elementary education. The time of treatment for hemodialysis was 5 to 9 years, with $52 \%$ the main underlying disease being Systemic Arterial Hypertension (SAH). Regarding the importance of dialysis, everyone considers treatment important. Conclusion: These data helped health professionals to know the profile of patients to help plan care aimed at prevention and also to improve the life expectancy of those who undergo hemodialysis.
\end{abstract}

Keywords: Renal Dialysis; Chronic Kidney Disease; Health Profile.

I Médico Veterinário, Doutor em Ciência Animal pela Universidade Federal do Piauí - UFPI, Professor Adjunto da Universidade Estadual do Piauí - UESPI. E-mail: franciscoaraujo@ccs.uespi.br Teresina - PI

2 Graduada em Enfermagem pelo Centro Universitário do Piauí - UNIFAPI. E-mail: chicaovet@gmail.com Teresina - PI

3 Graduado em Enfermagem pelo Centro Universitário de Ciências e Tecnologia do Maranhão - UniFacema. E-mail: wendersoncosta09@hotmail.com Caxias - PI 4 Graduando em Tecnólogo em radiologia pelo Centro Universitário de Ciências e Tecnologia do Maranhão - UniFacema. E-mail: biielpedro707@gmail.com Caxias - MA 5 Graduada em Enfermagem pelo Centro Universitário de Ciências e Tecnologia do Maranhão - UniFacema. E-mail: brunnamattos4@gmail.com Caxias - MA 6 Graduada em Enfermagem pelo Centro Universitário de Ciências e Tecnologia do Maranhão - UniFacema. E-mail: biancasoares I922@gmail.com Caxias - MA 7 Graduado em Enfermagem pelo Centro Universitário de Ciências e Tecnologia do Maranhão - UniFacema. E-mail: rogeriocruz82@yahoo.com.br Caxias - MA 8 Graduando em Enfermagem pelo Centro Universitário de Ciências e Tecnologia do Maranhão - UniFacema. E-mail: eduzinhobds@gmail.com Caxias - MA 9 Graduando em Enfermagem pelo Centro Universitário de Ciências e Tecnologia do Maranhão - UniFacema. E-mail: wallison-lima I2@hotmail.com Caxias - MA 10 Graduanda em Enfermagem pelo Centro Universitário de Ciências e Tecnologia do Maranhão - UniFacema. E-mail: mariagabrielle980@gmail.com Caxias - MA II Graduando em Enfermagem pelo Centro Universitário de Ciências e Tecnologia do Maranhão - UniFacema. E-mail: amarildocaxias20@gmail.com Caxias - MA 12 Graduada em Enfermagem pelo Centro Universitário de Ciências e Tecnologia do Maranhão - UniFacema. Email: lisyanenathalia6@gmail.com Caxias - MA 


\section{INTRODUÇÃO}

O sistema renal é fundamental para a manutenção e equilíbrio do organismo humano. As principais funções dos rins é filtrar o sangue para controlar a quantidade de água e de sal do corpo e eliminar toxinas. Por ter inúmeras funções, é importante que os rins estejam saudáveis. Com a perda de alguma dessas funções acabam ocasionando as chamadas doenças renais, considerada um grande problema de saúde pública, por conta do crescente aumento de casos. Além disso, tem um impacto negativo sobre a qualidade de vida relacionada a saúde dos pacientes ${ }^{(1)}$.

A insuficiência renal é uma condição na qual os rins perdem a capacidade de realizar suas funções básicas, ocorrendo uma perda parcial da função renal, de forma lenta, progressiva. Calcula-se que a Doença Renal Crônica (DRC) atinge pessoas de todas as idades, mas principalmente os idosos. A insuficiência renal pode ser aguda, quando ocorre súbita e rápida perda da função renal; ou crônica, quando a perda é lenta, progressiva e irreversível. Em geral, é o resultado final da destruição tecidual e dos néfrons que causam lesões renais irreversíveis ${ }^{(2)}$.

Dentre as principais causas de morte da DRC está a Hipertensão Arterial Sistêmica (HAS), pois o rim é o órgão responsável pelo controle da pressão arterial; o diabetes mellitus que após anos também acarreta problemas renais; e glomerulonefrite resultado de uma inflamação nos rins. Para tais problemas a importância hemodiálise dá-se a promover a retirada de substâncias tóxicas, sais minerais e água do organismo denominada de Terapia Renal Substitutiva (TRS) com o auxílio de uma máquina ${ }^{(3)}$.

A hemodiálise faz o trabalho dos rins quando o mesmo está doente e não pode fazer. $O$ procedimento é através de uma máquina que libera o corpo dos resíduos prejudiciais à saúde, como o excesso de sal e de líquidos. Nos casos em que os remédios não são suficientes e a doença progride, pode ser necessário iniciar a hemodiálise, ela é indicada para pacientes com insuficiência renal aguda ou crônica grave ${ }^{(4)}$.

O número de pacientes com DRC que precisaram de diálise cresceu de 42 mil, em 2000, para 122 mil no ano de 2016. Já transplantados de rim no país só no ano passado foram, 5,7 mil pessoas, quantidade que vem aumentando, em média, $10 \%$ de um ano para o outro. $O$ Sistema Único de Saúde (SUS) foi responsável por 83\% das diálises feitas em $2016^{(5)}$.

A DRC consiste em lesão renal e perda progressiva e irreversível da função do rim. Em sua fase mais avançada, os rins não conseguem mais manter a normalidade do meio interno do paciente. $O$ enfermeiro a partir da compreensão desse estudo terá uma noção do perfil clínico e sociodemográfico dos pacientes com DRC, e com isso poderá planejar estratégias para $\circ$ tratamento a esses pacientes de uma maneira que ajude a amenizar as limitações e melhorar a qualidade de vida desses pacientes.

Por fim, tende a contribuir para compreensão clínica da realidade vivida pelo portador de DRC. E por ser uma doença que a cada ano tem um aumento considerável de pacientes que fazem hemodiálise. Os profissionais de saúde ao terem conhecimento sobre o perfil clínico de pacientes hemodialisados poderão contribuir na prevenção ou na identificação desses pacientes.

O problema abordado nesse estudo foi qual o perfil clínico e epidemiológico dos pacientes de um centro de hemodiálise,? e o principal objetivo dessa pesquisa visa levantar o perfil clínico e sociodemográfico de pacientes em um centro de hemodiálise trazendo as principais patologias de base, a faixa etária acometida, sexo e condições sociodemográficas que acometem os pacientes hemodialisados e reconhecer qual a importância da terapêutica dialítica para esses pacientes.

\section{MÉTODO}

Esta pesquisa trata-se de um estudo descritivo, exploratório, com abordagem quantitativa.A abordagem quantitativa é adequada à realização desse trabalho pelo fato de seu objeto de pesquisa ser um fenômeno mensurável e por se tratar de um tema que exige objetividade dos seus resultados.

A pesquisa foi realizada no Centro de Hemodiálise localizado no município de Teresina-PI. Está localizada em um condomínio que abrange outras clinicas com várias especialidades diferentes. É um centro privado, mas que também realiza atendimento pelo Sistema Único de Saúde (SUS).

No Período da pesquisa, o serviço atendia 247 pacientes no programa de hemodiálise. $O$ centro de hemodiálise tem o funcionamento das $07 \mathrm{~h}$ às $20 \mathrm{~h}$, de segunda a sábado e é dividido em três turnos. Conta com $42 \mathrm{ca}-$ deiras de hemodiálise, sala de urgência, recepção e sala para consulta.

As amostras foram formadas de forma casuística por aqueles indivíduos que estavam no ambiente do Centro de Hemodiálise obedecendo os aspectos éticos e os critérios de inclusão/exclusão.

Os critérios de inclusão foram: pacientes maiores de 18 anos e de ambos os sexos, portadores de DRC sob tratamento no Centro de Hemodiálise na cidade de Teresina - PI, que estavam realizando as sessões de hemodiálise ativamente há um mês, e que aceitaram participar da pesquisa, assinando o Termo de Consentimento Livre e Esclarecido (TCLE). Os critérios de exclusão foram: pacientes que estavam realizando tratamento há menos de um mês, que seja menor de idade, que não estavam aptos a responder o questionário.

Foi realizado a coleta com os pacientes do turno da 
manhã totalizando 50 indivíduos obedecendo os critérios de inclusão e exclusão.

Os dados da pesquisa foram coletados dos pacientes em hemodiálise através de um questionário disponibilizado em um estudo desenvolvido por Ferreira ${ }^{(6)}$ e Melo ${ }^{(7)}$ contendo questões sobre o perfil clínico e sociodemográfico. Essas questões abordaram os dados sociodemográficos e as patologias mais frequentes que acometem a qualidade de vida levando ao tratamento. Foram aplicadas aos pacientes enquanto estavam no Centro de Hemodiálise, em sessões de hemodiálise. A coleta dos dados da pesquisa foi realizada nos meses de outubro e novembro de 2019.

As análises dos dados foram feitas através da análise de variância e apresentados em números relativos e absolutos, da forma que melhor demonstrasse a quantidade amostral e análise das respostas dos sujeitos. Os dados foram digitalizados e armazenados em planilha do programa Microsoft Office Word 2013. Os resultados finais foram apresentados em tabelas.

Com os referidos dados analisados e com os resultados obtidos foi comparado com estudos já publicados em relação a essa temática, e que em sua maioria demostraram resultados semelhantes.
Por se tratar de um estudo envolvendo seres humanos, o projeto desta pesquisa foi enviado ao Comitê de Ética e Pesquisa (CEP) da Universidade de Pesquisa (UNIP) e recebeu aprovação sob o protocolo de Certificado de Apresentação para Apreciação Ética (CAAE) n ${ }^{\circ}$ |73788|9.7.0000.55|2.

A participação no estudo foi voluntária e sem identificação dos participantes. Estes, foram esclarecidos previamente sobre a finalidade da pesquisa e a desistência em participar do estudo, e aqueles que concordaram em participar foram convidados a assinar o TCLE.

O orientador e orientandos, respectivamente, comprometeram-se com as normas preconizadas pela Resolução CNS n $466 / 2012$ e suas complementares, que tratam dos aspectos éticos envolvendo seres humanos.

\section{RESULTADOS}

A Tabela 0 I a seguir apresenta o perfil sociodemográfico dos participantes do estudo, pode-se identificar a predominância do sexo masculino (80\%). A faixa etária variou com maior predominância entre 48 a 57 anos e 58 a 67 anos (26\%). Quanto à cor da pele, a maioria considerava-se pardos (72\%); já o estado conjugal $66 \%$ eram casados. Em relação a renda familiar foi identificada nos

TABELA 1 - Variáveis sociodemográfica dos pacientes hemodialisados. Teresina, PI, Brasil 2019.

\begin{tabular}{|c|c|c|}
\hline Sexo & $n$ & $\%$ \\
\hline Masculino & 40 & 80 \\
\hline Feminino & 10 & 20 \\
\hline \multicolumn{3}{|l|}{ Faixa etária (anos) } \\
\hline $18-27$ & 2 & 4 \\
\hline $28-37$ & 1 & 2 \\
\hline $38-47$ & 8 & 16 \\
\hline $48-57$ & 13 & 26 \\
\hline $58-67$ & 13 & 26 \\
\hline $68-77$ & 11 & 22 \\
\hline $78-87$ & 2 & 4 \\
\hline \multicolumn{3}{|l|}{ Cor/Raça } \\
\hline Branco & 3 & 6 \\
\hline Amarelo & 4 & 8 \\
\hline Pardo & 36 & 72 \\
\hline Negro & 6 & 12 \\
\hline Indígena & 1 & 2 \\
\hline \multicolumn{3}{|l|}{ Estado Conjugal } \\
\hline Casado & 33 & 66 \\
\hline Solteira & 7 & 14 \\
\hline Divorciado & 3 & 6 \\
\hline Viúvo & 4 & 8 \\
\hline União estável & 3 & 6 \\
\hline \multicolumn{3}{|l|}{ Ocupação } \\
\hline Aposentado & 39 & 78 \\
\hline Trabalhando & 1 & 2 \\
\hline Desempregado & 5 & 10 \\
\hline Autônomo + Aposentado & 5 & 10 \\
\hline \multicolumn{3}{|l|}{ Renda Familiar } \\
\hline Até 1 salário & 41 & 82 \\
\hline 1 a 3 salário & 7 & 14 \\
\hline 3 a 5 salário & 1 & 2 \\
\hline Nenhum salário & 1 & 2 \\
\hline Total & 50 & 100 \\
\hline
\end{tabular}


participantes até I salário mínimo (82\%); e maior predominância de aposentados (78\%).

$\mathrm{Na}$ Tabela 2 mostra a prevalência de que a maioria dos participantes tem procedência de Teresina (44\%), $12 \%$ sendo do Maranhão e uma variedade de participantes de outras cidades vizinhas do Piauí.

Em relação à escolaridade, houve predomínio dos pacientes nos quais não completaram o ensino fundamental (34\%), seguido de ensino médio completo (26\%), $18 \%$ sem escolaridade, $12 \%$ ensino fundamental completo, respectivamente. Quanto ao tempo que os participantes fazem hemodiálise, o resultado obtido foi de 5 a 9 anos.

$\mathrm{Na}$ análise clínica mostrou que a doença de base mais prevalente foi Hipertensão Arterial Sistêmica (HAS) $(18 \% /)$, seguido de diabetes mellitus ( $10 \%)$, entretanto, vale destacar que $52 \%$ dos pacientes não souberam informar a doença de base que desencadeou a DRC, conforme demostrado na Tabela 03.

Ao serem questionados os participantes sobre a importância da hemodiálise para o tratamento todos alegaram que era de muita importância na vida deles. Ao afirmarem isso relatavam que sem a hemodiálise não poderiam viver com os seus familiares e não teriam uma qualidade de vida aceitável, mesmo com as limitações do tratamento. Alegavam que não gostavam de estar naquelas condições, em relação ao fato de não poderem mais trabalhar ou o fato da mudança de alimentação, mas que era o melhor para o seu bem-estar e que poderiam realizar as suas atividades diárias normalmente.

\section{DISCUSSÃO}

Em relação ao sexo dos participantes, grande parte era do sexo masculino ( $80 \%$ ) e $10 \%$ feminino. Esse estudo corrobora com as literaturas encontradas em que o estudo de Silva e colaboradores ${ }^{(8)}$ o sexo masculino se apresenta em maior proporção. Isso se dar pelo fato de que eles têm menor cuidado preventivo em relação a saúde e buscam os serviços de saúde quando as morbidades já se encontram em nível mais avançado(9).

Quanto a faixa etária, o estudo mostrou que 38 a 47 anos foi de $16 \%$, 68 a 77 anos foi de $22 \%$. E com a maior prevalência foi de $26 \%$ dos pacientes que possuíam idade entre 48 a 57 anos e 58 a 67 anos, no qual demostraram resultados semelhantes no estudo de Oliveira e colaboradores $^{(10)}$, em que foi realizado em 74 pacientes no qual $20,27 \%$ possuíam idade entre 48 a 57 anos e 58 a 67 anos.

TABELA 2 - Procedência dos pacientes hemodialisados. Teresina, PI, Brasil, 2019.

\begin{tabular}{|c|c|c|}
\hline Procedência & $\mathbf{N}$ & $\%$ \\
\hline Teresina & 22 & 44 \\
\hline União & 4 & 8 \\
\hline José de Freitas & 3 & 6 \\
\hline Maranhão & 6 & 12 \\
\hline Piripiri & 1 & 2 \\
\hline Barras & 1 & 2 \\
\hline Água Branca & 2 & 4 \\
\hline Lagoa Alegre & 1 & 2 \\
\hline Palmerais & 1 & 2 \\
\hline Elesbão Velozo & 3 & 6 \\
\hline Miguel Alves & 2 & 4 \\
\hline Alto Longá & 1 & 2 \\
\hline Beneditinos & 1 & 2 \\
\hline Demerval Lobão & 1 & 2 \\
\hline Nazária & 1 & 2 \\
\hline Total & 50 & 100 \\
\hline
\end{tabular}

Legenda: $\mathrm{N}=$ número; $\%$ = percentual.

Fonte: Dados da Pesquisa, 2019.

TABELA 3 - Doenças de base dos pacientes hemodialisados. Teresina (PI), Brasil, 2019.

\begin{tabular}{|c|c|c|}
\hline Doenças de Base & $\mathbf{N}$ & $\%$ \\
\hline Hipertensão Arterial Sistêmica & 9 & 18 \\
\hline Diabetes Mellitus & 5 & 10 \\
\hline Lúpus Eritematoso Sistêmico & 1 & 2 \\
\hline Doença Renal Policística & 2 & 4 \\
\hline Causa Genética & 1 & 2 \\
\hline Hipertensão Arterial Sistêmica + Diabetes Mellitus & 3 & 6 \\
\hline Não souberam informar & 26 & 52 \\
\hline Outros & 3 & 6 \\
\hline Total & 50 & 100 \\
\hline
\end{tabular}

Legenda: $\mathrm{N}=$ número; $\%$ = percentual.

Fonte: Dados da Pesquisa, 2019. 
A cor da pele a prevalência maior foi da cor parda (72\%). Já em outro estudo(8) houve a prevalência da cor branca $(93,1 \%)$, e no de Santos e colaboradores ${ }^{(11)}$ a prevalência foi da cor negra (40,5\%), discordando desse estudo.

Com relação ao estado conjugal, $66 \%$ eram casados e $14 \%$ eram solteiros. $O$ companheirismo representa em grande maioria para esses pacientes como um papel de "cuidador" ou "acompanhante" dependendo do grau em que esse paciente se encontra em decorrência da doença ${ }^{\text {(II). }}$

Quando questionados sobre a ocupação que tinham naquele momento, 10\% disseram estar desempregados, e $10 \%$ eram autônomos, mas aposentados. A ocupação que mais se destacou com $78 \%$ foi a de aposentados, visto que os pacientes são submetidos a três seções de hemodiálise na semana alternadamente com duração de 4 horas, esse tratamento acaba os impondo a essa condição de vida. Já a renda familiar foi de até I salário mínimo com $82 \%$, essa baixa renda está associada com o fato que muitos não apresentam vínculo com trabalho e com isso dependem do auxílio do governo ${ }^{(12)}$.

Em relação a escolaridade, no estudo de Oliveira e colaboradores $^{(10)}$ (2017), foi observado que dos 74 pacientes entrevistados, $39 \%$ possuíam ensino fundamental incompleto, outro estudo teve resultados semelhantes no qual de 123 pacientes $68 \%$ possuíam ensino fundamental incompleto(13).

Esses dados são um alerta para os profissionais de enfermagem, pois os mesmos têm que fazer uso de uma linguagem mais popular evitando termos técnicos quando for dar orientações, cuidados ou educação para prevenção de complicações referentes ao tratamento para os pacientes ${ }^{(11)}$.

Em relação ao tempo de hemodiálise, estudos apontam que 40,76\% dos participantes analisados apresentavam tempo de hemodiálise de cinco ou mais anos, confirmando os dados desse estudo. A pesar de ser um tratamento muito importante, ao longo do tempo compromete a qualidade de vida dos usuários como restrições as atividades básicas do dia-a-dia, alterações nos hábitos de vida e diminuição social $\left.\right|^{(14,15)}$.

Ao serem abordados sobre a doença de base da DRC, $52 \%$ não souberam informar a verdadeira causa que os levaram a fazer hemodiálise, porém os que souberam informar, I8\% foi por hipertensão arterial sistêmica e 10\% diabetes mellitus. Em um estudo realizado no hospital público na cidade de Salvador relatou que 36,13\% corresponde a hipertensão arterial sistêmica, encontrandose em conformidade com os dados deste estudo ${ }^{(16)}$.

No estudo de Thomé e colaboradores ${ }^{(17)}$ as causas primárias mais frequentes da DRC foram hipertensão (34\%) e diabetes (31\%), seguido de glomerulonefrite crônica (10\%), rins policísticos (4\%), indefinidos e outros (10\%). Em relação a essas duas doenças hipertensão e diabetes fica claro que a principal prevenção para não chegar a DRC tem que ser iniciada e abordadas pelas Unidades Básicas de Saúde, para assim não se agravar. Em outra pesquisa sobre a causa da DRC nos participantes, $20 \%$ a causa foi por glomerulonefrite crônica, seguida de nefrite ( $17,5 \%)$, hipertensão (15\%), rins policísticos (I5\%), diabetes (5\%), e $20 \%$ não souberam responder ${ }^{18}$.

\section{CONCLUSÃO}

Diante dos dados descritos traçou-se um perfil sociodemográfico dos pacientes desta pesquisa, revelando a alta predominância de homens, adultos, de cor parda, procedência de Teresina. A ocupação aposentada com baixa renda de até um salário mínimo, que possuem apenas o ensino fundamental incompleto e a maioria eram casados.

No perfil clínico a predominância da doença de base que desencadeou a DRC foi a HAS, e fazendo tratamento a longo período de tempo entre 5 a 9 anos, do qual todos reconheceram o tratamento da hemodiálise com muita importância.

Os resultados encontrados dessa pesquisa em sua maioria são compatíveis com outros estudos da mesma temática já publicados, é importante fazer esse tipo de pesquisa para se manter atualizados sobre os pacientes que realizam hemodiálise, pois cada um dos dados coletados tem uma importância fundamental para a área da saúde.

Esses dados ajudaram os profissionais de saúde a conhecer o perfil dos pacientes para auxílio do planejamento de uma assistência direcionada à prevenção e também para melhorar a expectativa de vida dos que são submetidos a hemodiálise. 


\section{REFERÊNCIAS}

I. Brasil. Ministério da Saúde [homepage na internet]. Doenças renais: causas, sintomas, diagnóstico, tratamento e prevenção [acesso em 13 abr 2019]. Disponível em: https:// www.saude.gov.br/saude-de-a-z/doencas-renais.

2. Brasil. Ministério da Saúde [homepage na internet]. Você sabe o que é insuficiência renal? [acesso em I3 abr 2019]. Disponível em: http://www.blog.saude.gov.br/index.php/ promocao-da-saude/50376-voce-sabe-o-que-e-insuficiencia-real.

3. Brasil. Ministério da Saúde. Secretaria de Atenção à Saúde. Departamento de Atenção Especializada e Temática. Diretrizes Clínicas para o Cuidado ao paciente com Doença Renal Crônica - DRC no Sistema Único de Saúde. Brasília: Ministério da Saúde; 2014.

4. Sociedade Brasileira de nefrologia, organização [Internet]. São Paulo: Sociedade Brasileira de nefrologia; 2019; [acesso em 21 jul 2019]. Disponível em: https://sbn.org.br/publico/ tratatamentos/hemodialise/

5. Sociedade Brasileira de nefrologia, organização [Internet]. São Paulo: Sociedade Brasileira de nefrologia; 2017 (2016); [acesso em 14 abr 2019]. Disponível em: http://agenciabrasil.ebc.com.br/geral/noticia/2017-06/pacientes-com-doenca-renal-cronica-triplicam-em- I6-anos-no-brasil

6. Ferreira, LG. Caracterização sociodemográfica, clínica, psicossocial e espiritual de pacientes renais crônicos [dissertação]. São José do Rio Preto: Faculdade de Medicina de São José do Rio Preto; 2017.

7. Melo BTN. Avaliação da qualidade de vida relacionada à saúde de pessoas com insuficiência renal crônica em hemodiálise no município de João Pessoa-PB [dissertação]. João Pessoa - PB: Universidade Federal da Paraíba; 2018.

8. Silva F, Bettinelli LA, Bortoluzzi EC, Doring M, Fortes VLF, Dobner T. Terapia renal substitutiva: perfil sociodemográfico e clínico laboratorial de pacientes de um serviço de hemodiálise. Revista de Enfermagem [Internet]. 2017 [acesso em 23 ago 2019]; II(9):3338-3345. Disponível em: https:// pdfs.semanticscholar.org/ce42/9d0987040 I db333084a9f8d7ab47d3cdea I2.pdf.

9. Brasil. Ministério da Saúde. Política Nacional de Atenção Integral à Saúde do Homem: princípios e diretrizes. Brasília: Ministério da Saúde; 2008. Disponível em: http://bvsms.saude.gov.br/bvs/publicacoes/politica_nacional_atencao_homem.pdf.

10. Oliveira DPS, Lopes MLH, Silva GAS, Sousa SMA, Dias RS, Silva LVM. Perfil socioeconômico e clínico dos pacientes em programa hemodialítico. Revista de Enfermagem UFPE [Internet]. 2017 [acesso em jul 20I9] II(II):4607-46I6. Disponível em: https://pdfs.semanticscholar.org/59e3/ 2c6I53a237034f793b7e3c536ca I 2I 5a06de.pdf.

I I. Santos RR, Formiga LMF, Oliveira EAR de, Lima LHO,Araújo AKS, Santos BB de. Qualidade de vida de pacientes com insuficiência renal crônica sob tratamento hemodialítico. Revista Interdisciplinar [Internet]. 2015 [acesso em $23 \mathrm{fev}$ 2019] 8(3):83-92. Disponível em: https://revistainterdisciplinar.uninovafapi.edu.br/index.php/revinter/article/view/7/5/ pdf_238.

12. Kusumota L. Avaliação da Qualidade de Vida Relacionada à Saúde de Pacientes em Hemodiálise [Tese]. Ribeirão Preto: Universidade de São Paulo; 2005.

13. Valcanti CC, Chaves ÉCL, Mesquita AC, Nogueira DA, Carvalho EC de. Coping religioso/espiritual em pessoas com doença renal crônica em tratamento hemodialítico. Rev. esc. enferm. USP [Internet]. 2012 [acesso em 24 set 2019]; 46(4): 838-845. Disponível em: http://www.scielo.br/scielo.php?script=sci_arttext\&pi$d=S 0080-623420 I 2000400008 \&$ Ing=en. https://doi. org/I0.I590/S0080-623420I2000400008.

14. Ferreira RC, Silva Filho CR da. A qualidade de vida dos pacientes renais crônicos em hemodiálise na região de Marília, São Paulo. J. Bras. Nefrol. [Internet]. 20I I [acesso em I4 fev 2019]; 33(2):129-135. Disponível em: http://www.scielo.br/scielo.php?script=sci_arttext\&pi$\mathrm{d}=\mathrm{SO}$ I $0 \mathrm{I}-280020 \mathrm{I} 1000200003 \& \mathrm{lng}=\mathrm{en}$. https://doi. org/I0.1590/S0I0I-280020II000200003.

15. Madalosso FD, Mariotti MC. Terapia Ocupacional e qualidade de vida de pessoas com insuficiência renal crônica em hemodiálise. Cad Ter Ocup UFSCar. [Internet]. 2013 [acesso em 24 de ago 2019]; 2 I [3]:5 I I-520. Disponível em: http://www.cadernosdeterapiaocupacional.ufscar.br/index. php/cadernos/article/view/91 I/463.

16. Almeida MIC, Cardoso MS, Garcia CPC, Oliveira JRF de, Gomes MLF. Perfil dos pacientes renais crônicos de um Hospital Público da Bahia. Revista Enfermagem Contemporânea [Internet]. 2013 [acesso em 14 mar 2019]; 2(I): 157-|68. Disponível em: https://www5.bahiana.edu.br/index.php/enfermagem/article/download/290/225

17. Thomé FS, Sesso RC, Lopes AA, Lugon JR, Martins CT. Inquérito Brasileiro de Diálise Crônica 2017. J. Bras. Nefrol. [Internet]. 2019 [acesso em I3 jul 20I9]; 4I(2):208-2I4. Disponível em: http://www.scielo.br/scielo.php?script=sci_arttext\&pid=SO I O I =280020 I 9000200208-\&lngpt. Epub 28-Mar-2019. https://doi.org//0.1590/2/758239-jbn-2018-0178.

18. Higa K, Kost MT, Soares DM, Morais MC de, Polins BRG. Qualidade de vida de pacientes portadores de insuficiência renal crônica em tratamento de hemodiálise. Acta paul. enferm. [Internet]. 2008 [acesso em 24 de jul 2019]; 2 I (spe): 203-206. Disponível em: http://www.scielo.br/scielo.php?script=sci_arttext\&pid=S0 I 03=2 $10020080005000 \mathrm{I}$ 2-\&lngen. https://doi.org/I0.1590/S0 I03-210020080005000I2.

Recebido: 2020-02-28

Aceito: 2020-07-14 